\title{
Scientific Consciousness and Exploration Spirit in Shan Hai Jing
}

\author{
Xi Chen ${ }^{1, a}$ \\ ${ }^{1}$ Northwest Normal University, Lanzhou, Gansu, 730070
}

Keywords: "Shan Hai Jing"; Ancestors of the Times; Nature; Scientific Exploration;

\begin{abstract}
Shan Hai Jing" is an ancient Chinese book between the Warring States to the Qin and Han dynasties which more than two thousand years ago, there are a total of 18 books, scholars have paid great attention to its mythological value research since the Ming and Qing. The last century 80 years later, some scholars re-discussed the realistic color of "Shan Hai Jing". The book contains the people's study and records of natural resources at pre-Qin period, it not only objectively reflects the survival status quo of the ancestors, but also reflects the human instincts of curiosity, curiosity and the spirit of scientific exploration from the pragmatism brought to the nature of real life. "Shan Hai Jing" uses natural resources to describe a different way based on different observation purposes. In the classification of natural resources, it embodies certain scientific thoughts and enlightens the development and research of natural science in later generations.
\end{abstract}

\section{Introduction}

"Shan Hai Jing" is a very special book of Chinese pre-Qin Dynasty books, such as Yuan Ke said, "My ancient books, the most of the world's most mysterious Qi, Mo Shan Hai Jing. Shanhai by the bandit special history of the right, it is also the myth of the government ". Since the Han Dynasty, scholars believe that the "Shan Hai Jing" is a practical geography book, side material, but the Ming Dynasty Hu Yinglin "less room mountain pencil" on the views of predecessors questioned that this book is "ancient and modern language strange ancestors" Since then, scholars are more and more attention to the "Shan Hai Jing" in the strange color. "The study of Chinese mythology" proposed "Shan Hai Jing" "non-geographical books, non-fiction, as 'myth', is the accumulation of knowledge of the early people, including the early people of the cosmology, religious thought, moral standards, the early history of national history and the understanding of nature, etc. ", of course," Shan Hai Jing "there are many strange records, eccentric people and creatures for us to spread a bizarre picture. But it is undeniable that the "mountain" Although some animals and plants have a strange appearance, the human has a magical effect, but carefully scrutinized, which contains the ancestors of the nature of the exploration, which is the survival of mankind The environment of curiosity, but also reflects the human nature of the original exploration and awareness.

\section{The Reflection of Living Conditions of the Ancestors in the "Shan Hai Jing"}

Pre-Qin period social productivity and production level is relatively low and people rely mainly on the survival of nature. Compared with other pre-Qin Dynasty books, "Shan Hai Jing" describes the nature of the "Book of Songs", "Songs of Chu" to express the fun of fun, but as a study object, starting from their own needs, in the available resources for some practical investigation. Therefore, for the Ming Dynasty that the "Shan Hai Jing" is a geography book, museum view, we cannot deny, we should not only see the "Shan Hai Jing" in the mythological color, but also from the perspective of myth to explore it give us the endless traces of the survival of the ancestors. 
Shan Hai Jing by the mountain" to the landscape for the latitude and longitude, straightforward records of the mountains contain the resources involved in ore, water and biological. Ore mainly includes metal and nonmetallic, creatures include plants and animals.

Through the records of the ore, we can have an understanding of the level of industrial and commercial development in the era of the ancestors. "Shan Hai Jing" In addition to gold, silver special attention, but also concerned about the copper, iron. The record of non-metallic than the metal to know more detailed. "Jade is divided into twenty kinds of stone as many as forty, which shows that since the Stone Age, people have accumulated a wealth of knowledge about jade and stone, but also shows that even to bronze or iron as the main tool for labor Signs of the times, jade and stone is still a lot of use, "' Nanshan by the mountain of the mountain, "recorded in a plant can be blood jade. Guo Pu injection of blood jade "that can be used for jade luster." The first people with this plant juice will be dyed bright colors, so that jade looks more beautiful. "Xishan by the birds and birds with the mountains" in the fish can be "raw beads jade", indicating that people not only have a wealth of jade knowledge, and active mining jade, jade processing activities.

"Shan Hai Jing" on the ancestors of the main life production activities is also reflected. "Shan Hai Jing" in a lot of animals and plants to help people add physical fitness, so that the human body more robust. For example, "the Western Hills" in a plant of the fruit, people can eat after eating a sense of fatigue, there is a plant called the wood, its fruit can make people more strength. In addition, there are many animals and plants that can perform similar functions. In summary, these features include two categories, one is "food is not hungry" this can be wrapped belly, reduce the human hunger and animal plants; the other is to achieve "food", "food Force "," food without lying "," good food "and other effects of animals and plants. From which we can see the ancestors desire to have a more robust body to deal with various production activities. There are like the use of Mang grass poisoning feeding fish, the eradication of rodents and other information can be glimpse of the ancestors of life.

"Shan Hai Jing" recorded in the medical resources revealed at the time of the medical level. "Shan Hai Jing" in the medical capacity of resources with minerals, animals and plants, but the proportion of plants and animals greater. "Shanhai Jing" recorded in the animal and plant drugs a total of 70 cases, including 21 cases of botanical drugs, animal medicine, 49 cases, involving diseases are: deaf, scabies, hemorrhoids, wax, mad, warts, gangrene, carbuncle, vomit, white Ringworm and other 34 kinds, there are a variety of diseases such as: swollen disease, heartache, head, pain, abdominal pain, crazy disease, disease, abdominal disease, confidant disease Luo Ruihe "<Shan Hai Jing> sick test" in the "Shan Hai Jing" in the emergence of more than 40 kinds of disease name, which includes the above-mentioned various disease names, and made a detailed explanation. According to the modern medical division, these diseases are included in the surgery, internal medicine, ophthalmology and neurology, the most common of which the skin disease, in addition to the ancestors are often suffering from the nightmare.

It is easy to summarize these illnesses from life, but it is not a simple job to find the medicinal properties of animals and plants that apply to the corresponding disease. There may be some occasional factors, but more should be forced by the disease to the body to bring unbearable pain, prompting them to find ways to relieve the pain, the treatment of disease. Similar to the "Shen Nong tasting Baicao" example, the ancestors in order to determine the animal and plant contains the drug effect should be after a number of attempts, and sometimes in the absence of the situation may also inevitably take the chances of taking some rare of the animals and plants, just as people say "dead horse when the horse" mentality. In addition, there may be some accidental factors that prompted them to find the efficacy of a certain animal and plant. 
In general, the "Shan Hai Jing" on the mineral, medicine and other resources in the records, faithfully reflects the survival status quo of the ancestors and the spirit of science exploration.

\section{The Scientific Observation of Animals and Plants in the "Shan Hai Jing"}

Regardless of the extent to which human society develops, nature is always dominated. "Shan Hai Jing" era of human want a better survival, it must be on their own side of the environment have a corresponding understanding. The scientific observation of the ancestors of nature in the Shanhai Jing is mainly embodied in two aspects.

First, the ancestors according to different perspectives to observe the nature, come to focus on different records, observation is very detailed. Some focus on the recording of mountain property resources, and some focus on the observation of a certain animal and plant, and some focus on writing some animal and plant unique habits and functions.

To animal and plant, for example, according to the ancestors recorded animal and plant information when the level of detail, we can "Shan Hai Jing" in the animal and plant records are divided into three categories.

The first category is only a simple description of the name, such as: stone crisp mountain and the wood more brown. This description only involves the name and genus, concise strokes, much like a record of local property records and so many scholars believe that "Wushan Mountain" is the nature of geographical books.

The second category on the basis of the first category increased the external observation of animals and plants. Such as: There is a beast Yan, like dolphins and white hair and like dolphins and white hair, as big as black and black, ("The Western Hills by the bamboo")

There are wood Yan. This type of description of animal and plant external image of the observation is very detailed. Animal description contains shape, sound, coat color, texture, and even the description of the shape and characteristics of hair. The plants are drawn from the overall size to the branches, from the leaf color to the texture shape.

The third type of animal and plant description more detailed, in addition to the previously mentioned shape, sound and other external description, but also talked about the characteristics of animals, rest habits and plant growth environment and animal and plant functions, such as:

There are fish Yan, its shape, such as cattle, mausoleum, snake tail wing, the feathers in the next and the sound of such as cattle, winter and resurrection. Eat without swollen disease. ("Nanshan by the mountain")

The grass has a Lai Lai, like a white leek, and was born in the stone, but also wood and health, food has been heartache. ("The Western Hills by the small mountain")

Ancestors noted that with "winter death and resurrection" of the characteristics, it is likely that this animal has hibernation habits, winter sting and spring out of activities. At that time, the concept of "hibernation" had not yet been formed, and the ancestors had discovered that the animals had disappeared in the winter, and the spring came out again, or saw that was as immortal as in the winter, "Winter death and resurrection" of the summary. The plant description in the above example also opens up a new focus, covering the growth environment and conditions. All of which reflect the human observation of animals and plants has been more detailed.

From the above three types of description can be seen, the ancestors of the flora and fauna is a reflection of diversity, there is no uniform standard, not simply mechanized records. The name may be due to the fact that such animals and plants are more common at the time and people are very familiar with, so no need to say. There may also be an official survey of local resources. Those detailed description of the appearance of animal and plant morphology of the information, observe 
the full, fine strokes, it may be rare animals and plants, accidentally discovered or infrequently encountered in the situation, the ancestors of its appearance was carefully, As the contemporary scientific investigation, found that the new species is always a detailed observation of the study, and the ancestors do not have the awareness and conditions of study, only to maximize the use of the naked eye to see, facial features Recorded, this is already a conscious scientific observation. The third type of animal and plant records also increased the habit of animal rest, which contains the scientific exploration of consciousness is very obvious. Ancestors for the existence of animals and plants exist not only recorded their appearance, but also their work and rest, action, habits, growth environment and so on a detailed study, a few figures described behind a long time accumulation and intentional observation. These three types of animals and plants are recorded as observers in their books, the records of animal and plant functions reflect the ancestors concerned to actively explore the relationship between animals and plants and their own.

Third, the "Shan Hai Jing" on the classification of natural resources"Shan Hai Jing" has a sense of classification, especially for the offspring of animal and plant classification science has enlightenment power.

"Shan Hai Jing" will be divided into gold ore, jade, stone three categories, the rest of rare metal copper, iron, tin and other single proposed. Gold has gold, red gold, platinum of the points, jade water jade, white jade, Cangyu, copper and other 20 categories, stone stone, Qing Shi, magnets and so on more than 40 kinds.

Animal and plant classification in this regard the performance of more obvious. "Shan Hai Jing" plant will be divided into two kinds of grass and wood, referring to plants are usually "grass Yan" "wood Yan" or "its grass more ... ..." "its wood ... ..." to the beginning, this more vivid description of the classification is that when there is no plant in the mountains or plant scarcity, it is described as "no vegetation", "more wood, no grass" or "no wood and more grass", through these examples we It is certain that the ancestors of the division of plants is "grass" and "wood" two categories. This classification method has great influence on the system of the descendants of medicine books. The method of "Zaoya", "Shen Nong's Materia Medica" and "Compendium of Materia Medica" are inheriting this classification method, and constantly improve the classification of animal and plant.

The classification of animals in the Shan Hai Jing is more complicated than that of plants, not only in many categories but also in the way of division. In the "overseas South" before the ancestors of the animals are divided into birds, beasts, fish, snakes and insects five categories. Its mark for the description of the animal when the first sentence on the animal species, such as "there is a beast ... ...", "there is a bird Yan ... ...", "more ... ... fish," but mentioned when the snake is another class said "there snake Yan", and then decorated with its shape, habits and other aspects of the description. If the animals on a mountain to give a brief description, sometimes called "is more birds, monsters, odd fish", "birds and animals Mo, is more than a snake.", sometimes called "more monsters, water and more strange fish. More viper, more strange snake ", which can be seen on the animal roughly divided into birds, beasts, fish, snakes, insects five categories. But in the "overseas south" "knot Hungary" in such a passage "since the mountain, insects for the snake, snake for the fish", since then, although the record is still birds, beasts, fish, snakes , Insect five categories, but its meaning has been changed.

From the "Shan Hai Jing" text, when people on the natural environment and the existence of resources to explore the consciousness and behavior, because the text in the interpretation of their own understanding of the phenomenon with the exaggeration, metaphor, association and other practices, resulting in "Shan Hai Jing" Weird colors, but these are built on the basis of objective reality. The article only from the "Shan Hai Jing" embodied in the sense of scientific exploration to 
start to understand the people's living conditions, but the "Shan Hai Jing" contains other disciplines of information is also a lot, such as geography, medicine, religion, etc., it can not only study for the myth The provision of materials, but also on the early development of other disciplines has a very important significance, it is worth our more in-depth and extensive research.

\section{References}

[1] Hao Yi Xing. "Shan Hai Jing Jian Shu" [M]. Bashu press, 1985

[2] Yuan Ke. "Shan Hai Jing Jing Note"[M]. Shanghai Ancient Books Publishing House, 1980.

[3] China "Shan Hai Jing" academic symposium editor. "Shan Hai Jing by the new exploration"[M]. Sichuan Academy of Social Sciences Press, 1986

[4] Xu Xianzhi."Shanhaiguan Jingyuan"[M]. Wuhan Publishing House, 1991

[5] Zhang Zhitian. "Introduction to the Shanhai Jing"[M]. Tianma Book Co., Ltd., 2003

[6] Lin Huixiang."Cultural Anthropology"[M]. Commercial Press, 1991

[7] Edward Taylor, Lian Shushen. "primitive culture"[M]. Guangxi Normal University Press, 2005 [8] James • Joe Fei, Lei Ze, Xu Yuxin, Wang Peiji, Zhang Ze Shi. "Jinzhi"[M]. popular literature and art press, 2009

[9] Levi • Boulevard, Ding You. "original thinking"[M]. The Commercial Press, 2009

[10] Ding Shan. "Ancient Chinese religion and mythology test"[M]. Shanghai Bookstore Publishing House, 2011 